\title{
Retinoids and proliferative verrucous leukoplakia (PVL). A preliminary study
}

\author{
Rafael Poveda-Roda ${ }^{1}$, Jose V. Bagán ${ }^{2}$, Yolanda Jiménez-Soriano ${ }^{3}$, Jose-María Díaz-Fernández ${ }^{1}$, Carmen \\ Gavaldá-Esteve ${ }^{1}$
}

${ }^{1}$ Staff physician. Service of Stomatology. Valencia University General Hospital

${ }^{2}$ Head of the Service of Stomatology. Valencia University General Hospital. Chairman of Oral Medicine, Valencia University Medical and Dental School

${ }^{3}$ Assistant Professor Doctor Valencia University. Valencia (Spain)

Correspondence:

C/ La Guardia Civil n 20 E5 pta 29,

46020 Valencia, Spain,

poveda@uv.es.

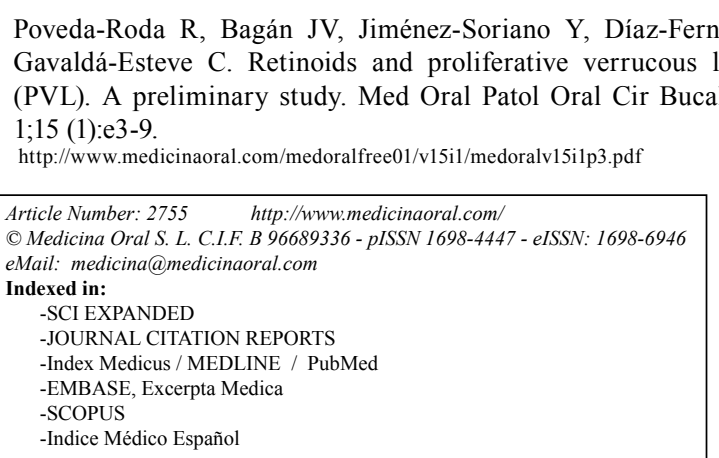

\begin{abstract}
Objective. A study is made of the efficacy and adverse effects of retinoid therapy applied to the white lesions of proliferative verrucous leukoplakia (PVL). Material and methods. The results of retinoid therapy were evaluated in 17 patients diagnosed with PVL. Topical retinoids were used in 5 patients, in the form of two daily applications of $0.1 \%$ 13-cis-retinoic acid in orabase for an average of 6.17+/-3.13 months. Systemic retinoids were used in 11 patients, with the administration of $25 \mathrm{mg}$ /day of acitretin in tablet form for an average of 5.41+/-2.02 months. One patient successively received the topical and systemic retinoid formulations. The course and results were evaluated on a blind basis by two investigators. The adverse effects of the medication were also assessed. Results. Clinical improvement was recorded for 7 lesions (38.8\%) (six involving systemic treatment and one as a result of topical application). Clinical worsening was recorded in the same proportion (5 lesions with systemic therapy and two with topical treatment), while four lesions (22.4\%) showed no changes (one lesion with systemic therapy and three with topical treatment). Adverse effects were documented in all the patients administered the systemic formulation, versus in only one patient administered topical retinoids. The most frequent problems were desquamation and pruritus. Conclusion. Although topical or systemic retinoic acid produces some improvement in about one-third of all patients with PVL, further studies are needed to assess the efficacy and safety of these products, in view of the important percentage of individuals who worsen despite therapy, and the frequent appearance of adverse effects.
\end{abstract}

Key words: Retinoids, retinoic acid, proliferative verrucous leukoplakia, adverse effects. 


\section{Introduction}

Proliferative verrucous leukoplakia (PVL) was first described by Hansen et al. in 1985 (1). It is characterized by the appearance in the oral cavity of white plaques in multiple locations and with different forms of presentation (homogeneous, patchy, verrucous) that progress towards oral squamous cell carcinoma in over $70 \%$ of all cases (2) - with the successive appearance of multiple primary tumors (a phenomenon known as "field cancerization") (3). The etiology of the process remains unclear, and the treatments used (analogous to those usually employed in the management of leukoplakia) appear to be scantly effective in controlling the disease - though few studies on the treatment of PVL can be found in the literature. Femiano et al. (4) used metisoprinol (inosine) associated to surgery, with good results, and better than those obtained with surgery alone. Among the treatments used, mention can be made of the $\mathrm{CO} 2$ laser, surgery, surgery associated to radiotherapy, cryotherapy, topical vitamins, bleomycin and photodynamic therapy with delta-aminolevulinic acid (ALA).

The present study was designed to assess the efficacy and adverse effects of systemic or topical retinoid therapy applied to the white lesions of PVL.

\section{Material and Methods}

The study was carried out in the Service of Stomatology of Valencia University General Hospital, with approval from the Clinical Research Committee and Ethics Committee of the center.

Following usual clinical practice, use was made of retinoids (as 13 -cis-retinoic acid $0.1 \%$ paste in orabase applied twice a day, or $25 \mathrm{mg}$ acitretin tablets -Neotigason ${ }^{\circledR}$ - once a day) in 17 patients diagnosed with PVL. Other potential study candidates were not included because they failed to follow regular therapy (5 patients), abandoned the treatment prematurely (6 patients), or because the photographic records failed to meet the requirements for adequate assessment of the lesions ( 2 patients). Of the 17 patients finally included in the study, 5 received treatment with $0.1 \% 13$-cis retinoic acid paste, 11 received $25 \mathrm{mg}$ acitretin tablets, and one successively received the topical and systemic retinoid formulations.

The necessary criteria for establishing the diagnosis of PVL were the following: simultaneous presence of several leukoplasic lesions in the oral cavity; distribution of the white lesions in different zones of the oral cavity, with the progressive occupation of large mucosal extensions; clinical follow-up of the lesions for at least two years or confirmation of progression to squamous cell carcinoma in cases with shorter follow-up, and histological confirmation of hyperkeratosis associated or not to variable degrees of epithelial dysplasia.

Women of child-bearing potential were not included in the study, due to the established teratogenicity of retinoids (category X of the United States FDA).

Photographic registries were obtained of all patients to assess the changes following treatment (i.e., response to the medication). We retrieved photographs of 18 white lesions in 17 patients diagnosed with PVL, obtained before and after treatment with retinoic acid.

We thus obtained 18 pairs of images (before treatmentafter treatment) corresponding to the 17 included patients (two pairs of images were obtained in one patient). The images were subjected to evaluation on a blind basis by two investigators, with a view to identifying changes (improvement or worsening), or the absence of change as a result of treatment (Figures 1, 2 and 3). Investigators must evaluate changes in size and appearance of lesions, specifically changes from homogeneous to verrucous or vice versa, and changes in colour. Changes in number of leukoplakias per patient were not evaluated. In cases of disagreement, the criterion of a third investigator was requested. If disagreement persisted among all three evaluators, the patient was removed from the study. The concordance between the two initial evaluators was required to be $\geq 80 \%$.

The patient clinical histories were likewise used to register the adverse effects occurring during treatment with any of the retinoid formulations. The evaluation of adverse effects was made among all patients who had received some dose of retinoids (topical or systemic) ( 25 patients), independently of whether they were also included in the image study group or not.

A descriptive statistical study was made (means and frequencies). Analytical statistics were not used, due to the presence of uncontrolled variables (severity or initial extent of the lesions, degree of dysplasia, previous carcinomas, etc.) that could have led to erroneous conclusions.

\section{Results}

The demographic and clinical dates of the patients are summarized in table 1.

The mean patient age was 61.44 years (range 43-85 years, standard deviation $-\mathrm{SD}- \pm 12.49)$. Males were somewhat less numerous than females $(41.2 \%$ versus $58.8 \%$ ). This proportion persisted on considering the total series of 30 patients in whom retinoic acid treatment in any of its forms had been indicated $(56.6 \%$ females and $43.3 \%$ males). Mean time from the estimated beginning of disease (PVL) to the start of treatment with retinoids was 77.5 months ( $\mathrm{SD} \pm 58.7$ months).

As has been commented, PVL has a marked tendency to develop oral squamous cell carcinoma over time. In our study, 5 patients had developed at least one such carcinoma: three patients had developed one lesion, one patient developed two, and another had developed as many as 5 successive carcinomas in different localiza- 


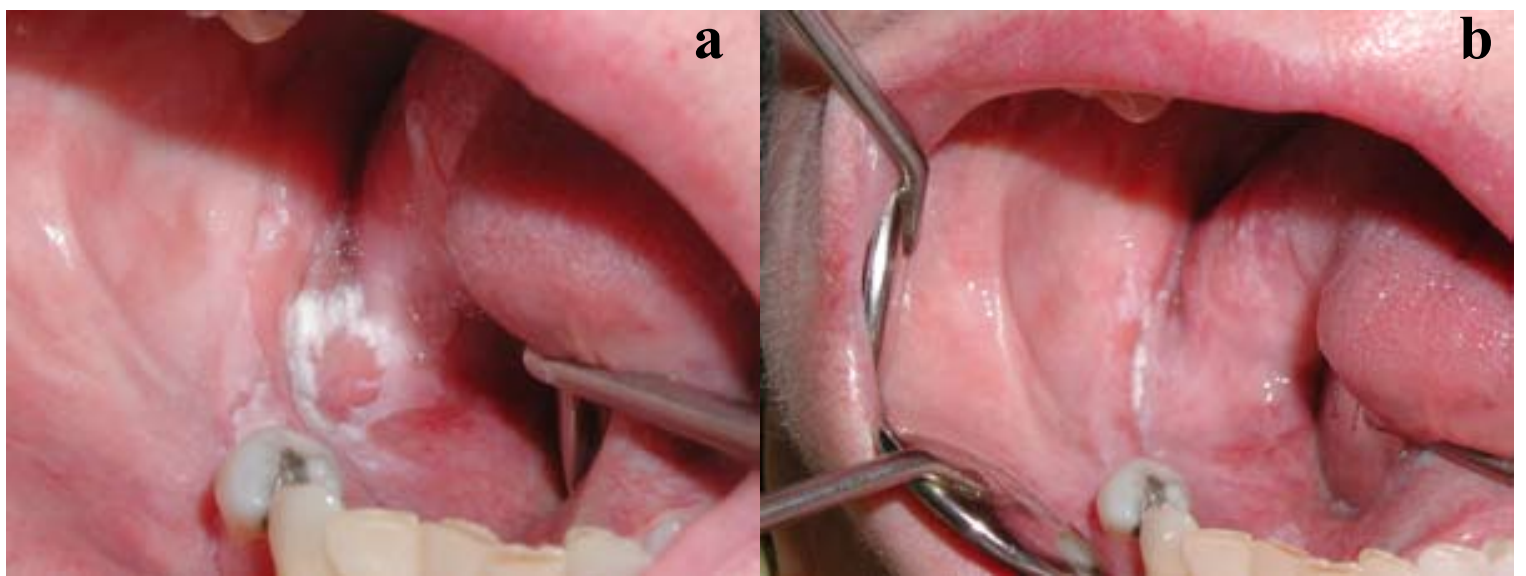

Fig. 1. Improvement after treatment with retinoids. a: before treatment. b: after treatment.

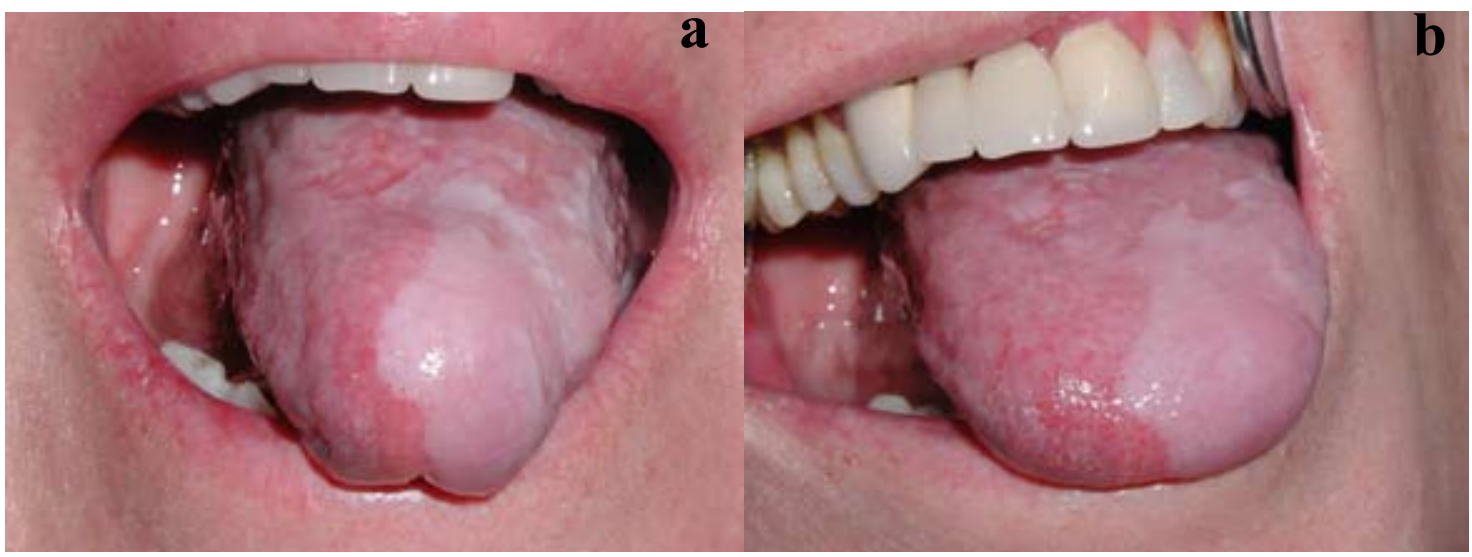

Fig. 2. Without changes after treatment with retinoids. a: before treatment. b: after treatment.

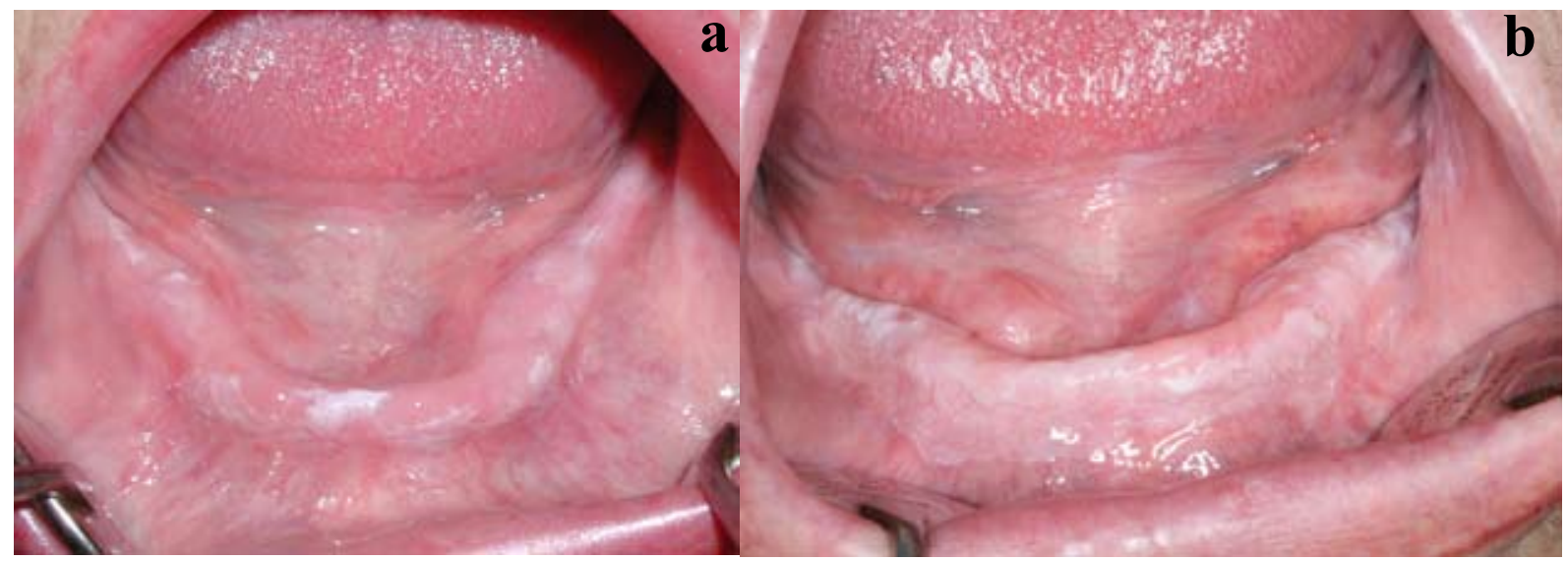

Fig. 3. Worsening after treatment with retinoids. a: before treatment. b: after treatment. 
Table 1. Data of the patients included in the evaluation of photographic registries.

\begin{tabular}{|c|c|c|c|c|c|c|c|c|c|}
\hline $\mathbf{n}$ & Age & Sex & $\begin{array}{l}\text { Prev. } \\
\text { treat. (a) }\end{array}$ & $\begin{array}{l}\text { Prev. } \\
\text { OSCC } \\
\text { (b) }\end{array}$ & $\begin{array}{l}\text { Diag. to } \\
\text { treat. (c) }\end{array}$ & $\begin{array}{l}\text { Treat. } \\
\text { (d) }\end{array}$ & $\begin{array}{l}\text { Dur. } \\
\text { treat. } \\
\text { (e) }\end{array}$ & $\begin{array}{l}\text { Objective im- } \\
\text { provement } \\
\text { (f) }\end{array}$ & Adverse effects \\
\hline 1 & 59 & $\mathrm{~F}$ & 12 & yes (2) & 139 & tabl. & 6 & no changes & $\begin{array}{c}\text { Desquamation, erythema, it- } \\
\text { ching. Increased cholesterol and } \\
\text { triglycerides }\end{array}$ \\
\hline 2 & 67 & M & 15 & yes (1) & 156 & tabl. & 9 & worse & $\begin{array}{l}\text { Hot flushes, photosensitivity. } \\
\text { Increased cholesterol and tri- } \\
\text { glycerides }\end{array}$ \\
\hline 3 & 66 & M & 1245 & yes (5) & 126 & tabl. & 8 & improved & $\begin{array}{l}\text { Fragile nails, alopecia, desqua- } \\
\text { mation, blurry vision }\end{array}$ \\
\hline 4 & 64 & $\mathrm{~F}$ & 56 & no & 76 & tabl. & 5 & worse & Dry eyes and ocular itching \\
\hline 5 & 49 & $\mathrm{~F}$ & 56 & no & 156 & tabl. & 4 & improved & Alopecia \\
\hline 6 & 74 & $\mathrm{~F}$ & no & yes (1) & 26 & pom. & 8 & worse & No \\
\hline 7 & 85 & $\mathrm{~F}$ & no & yes (1) & 60 & tabl. & 5 & improved & Pruritus \\
\hline 8 & 48 & $\mathrm{~F}$ & 5 & no & 68 & tabl. & 5 & worse & $\begin{array}{c}\text { Hyperglycemia, desquamation, } \\
\text { pruritus, alopecia }\end{array}$ \\
\hline 9 & 70 & $\mathrm{~F}$ & 35 & no & 56 & tabl. & 4 & worse & $\begin{array}{l}\text { Dropout due to rectal bleeding } \\
\text { and cramps }\end{array}$ \\
\hline 9* & 70 & $\mathrm{~F}$ & 35 & no & 60 & pom. & 6 & worse & No \\
\hline 10 & 73 & $\mathrm{M}$ & 5 & no & 24 & tabl. & 8 & improved & Alopecia \\
\hline 11 & 56 & M & 51 & no & 124 & tabl. & 2 & improved & Desquamation, bleeding, itching \\
\hline 12 & 45 & M & 51 & no & 24 & tabl. & 5 & worse & $\begin{array}{c}\text { Very intense desquamation, } \\
\text { pruritus }\end{array}$ \\
\hline 13 & 47 & $\mathrm{~F}$ & 5 & no & 144 & pom. & 4 & no changes & No \\
\hline 14 & 75 & $\mathrm{~F}$ & 5 & no & 35 & pom. & 11 & improved & Itching \\
\hline 15 & 52 & $\mathrm{M}$ & no & no & 17 & pom. & 2 & no changes & No \\
\hline 16 & 69 & $\mathrm{~F}$ & no & no & 24 & pom. & 6 & no changes & No \\
\hline 17 & 43 & $\mathrm{M}$ & 4 & no & 124 & tabl. & 4 & improved & $\begin{array}{l}\text { Desquamation, pruritus. In- } \\
\text { creased cholesterol and trigly- } \\
\text { cerides }\end{array}$ \\
\hline
\end{tabular}

(a) Previous treatment: 1 topical corticoids. 2 systemic corticoids. 3 topical immunosuppressors. 4 retinoic acid. 5 laser vaporization. 6. conventional surgery; (b) Previous oral squamous cell carcinomas (number shown in parenthesis); (c) Time in months from diagnosis of the disease to start of treatment with retinoids; (d) Type of treatment with retinoids: pom=paste, tabl=tablets; (e) Duration in months of treatment with retinoids; (f) Evolution of the lesions according to the evaluators. *The rows headed by the number 9 correspond to the same patient. OSCC $=$ oral squamous cell carcinoma.

Table 2. Adverse effects seen in the 25 patients treated with retinoids.

\begin{tabular}{|l|c|l|c|}
\hline \multicolumn{1}{|c|}{ Frequent } & No. of patients & \multicolumn{1}{c|}{ Isolated } & No. of patients \\
\hline Pruritus (itching) & 9 & Asthmatic crisis & 1 \\
\hline Desquamation & 8 & Rectal bleeding & 1 \\
\hline Alopecia & 5 & Hyperglycemia & 1 \\
\hline Dyslipidemia & 4 & Joint pain & 1 \\
\hline Fragile nails & 4 & Cramps & 1 \\
\hline Hot flushes / reddening & 3 & Dry mouth & 1 \\
\hline Blurry vision & 3 & Photosensitivity & 1 \\
\hline
\end{tabular}


tions of the oral cavity. All these carcinomas had developed previously to the introduction of treatments with retinoids. Two patients developed oral squamous cell carcinomas during the period in which they were receiving retinoic acid or in the period immediately after the end of treatment ( 3 months). These two patients had received systemic retinoids. The tumors were located in the cheek mucosa and left lateral margin of the tongue, respectively, and both were successive primary lesions: in one of the patients the tumor represented the second carcinoma, while in the other it corresponded to the third recorded lesion.

The mean duration of retinoid treatment was $5.41 \pm 2.02$ months in the case of the systemic retinoids, and $6.17 \pm$ 3.13 months for the topical retinoids. Globally, the mean duration of treatment was $5.67 \pm 2.37$ months (range 2-11 months). The mean time from initial diagnosis of the disease to the start of retinoid treatment was about 6.5 years (80.11 months, range $24-156, \mathrm{SD} \pm 51.06)$.

Concordance between the initial evaluators was $94 \%$. Only in one case the third evaluator was required. So, no patients were excluded from the study because of disagreement between evaluators. These investigators detected clinical improvement in $38.8 \%$ of the evaluated lesions. Worsening was documented in the same proportion of lesions, while four lesions (22.4\%) showed no changes after treatment. Of the 7 lesions that were seen to have improved, 6 had been treated with tablets and one with paste. As regards the lesions that worsened, 5 had been treated with tablets and two with paste. One of the lesions in which no changes were noted had been treated with tablets, and the other three with paste.

The most frequent adverse effects were skin desquamation and pruritus. Alopecia was also common, as were slight elevations in serum cholesterol and triglycerides, fragile nails, hot flushes and blurry vision. All the patients administered systemic retinoid treatment suffered some type of adverse effect, and four of them had to suspend the treatment as a result: two because of intense pruritus, one due to asthma crisis, and one because of rectal bleeding. In all four patients the symptoms disappeared after suspending the medication. The recorded adverse effects are shown in Table 2.

\section{Discussion}

Proliferative verrucous leukoplakia (PVL) manifests in the form of white lesions distributed over one or more locations within the oral cavity, and which are clinically and histologically indistinguishable from typical leukoplakia lesions. The disease can remain without change for years; it is therefore understandable that the treatments used for the white lesions of PVL are the same as those applied to leukoplakias. On the other hand, as a result of the refractory nature of the lesions, a broad range of therapeutic approaches known to have some ef- fect in patients with leukoplakia have also been used to treat PVL. However, very few publications in the literature have specifically addressed the treatment of PVL. The published series of patients diagnosed with PVL include smokers. Hansen, in the study that described the disease for the first time (1), reported that $62 \%$ of the patients consumed tobacco in one form or other. Zakrewska et al. (5) in turn reported a 70\% of smokers (7 of 10), while Silverman et al. (6) reported 31\%. Bagán et al. (7) reported the lowest presence of smokers (23.3\%). In our study, two of the 17 patients $(11.8 \%)$ were smokers (both were women), while four were ex-smokers (23.6\%) with a mean smoking cessation period of 9.75 years. All the patients in the study except two had received previous treatments for their oral lesions (laser, surgery or corticoids). In all of them the lesions failed to disappear or did not change despite treatment, or the lesions relapsed shortly after the end of treatment. This coincides with the observations of Hansen (1) who reported relapses despite treatment in 28 of 29 patients (radiotherapy, surgery, chemotherapy, multiple resections, laser, retinoids, other therapies). Silverman et al. (6), using surgery and surgery associated to radiotherapy, reported a percentage persistence of disease (live subjects with PVL or patients who died as a consequence of oral squamous cell carcinoma) of $85 \%$ after a mean follow-up of 11.6 years. These authors thus reported a $15 \%$ disease-free rate. Our series showed a greater female prevalence of PVL (56.6\% females and $43.3 \%$ males) - in coincidence with the observations of Hansen, Bagán Silverman and Cabey.

The primary objective of leukoplakia therapy should be the prevention of malignant transformation. Retinoids - which possess inhibitory action upon epithelial tissue proliferation and keratinization (8) - have been used in topical or systemic form for the treatment of leukoplakias. In our series we used 13-cis-retinoic acid for topical treatment and acitretin for sistemic treatment. Other retinoids as fenretinide were not considered in our study because it is not marketed nowadays in Spain. Hong et al. (9) employed 13-cis-retinoic acid in 24 patients with leukoplakia at a dose of $1-2 \mathrm{mg} / \mathrm{kg}$ /day during three months. They recorded improvement (lesion size reduction) in 16 patients $(67 \%)$ though there were relapses 2-3 months after treatment. In our series, 6 of the subjects treated improved of the disease, though 5 worsened despite of treatment, probably in relation to the progressive character of PVL. Silverman et al. (10) obtained complete resolution of the lesions in $44 \%$ of their patients using systemic vitamin A, while Sankaranarayanan et al. (11) reported the complete regression of precancerous lesions with systemic vitamin A in $52 \%$ of their patients. Although the improvement rates found by the authors are similar to our own (in the order of 50\% among the patients administered systemic retinoids), we 
consider it significant that none of the mentioned studies reported worsening of the lesions in spite of (or because of) the treatment provided. Our hypothesis is that worsening reflects the natural course (i.e., progression) of PVL rather than worsening because of treatment.

The results obtained on using topical retinoids for the treatment of leukoplakias, involving variable concentrations of tretinoin or isotretinoin in gel form $(0.05 \%$ to $0.1 \%$ ), are generally similar to those obtained with systemic retinoids. Shah (12) and Epstein et al. (13) obtained complete response in about $27 \%$ of their patients, with a partial response in 54\%. Boisnic et al. (14), using $0.1 \%$ retinaldehyde gel, observed the resolution of $17 \%$ of their leukoplakias, with improvement in $75 \%$. The use of higher concentrations of topical isotretinoin induced a significant reduction of the leukoplakia lesions without causing local or systemic adverse reactions (15). Of note is the fact that authors such as Piattelli et al. (16) obtained some degree of favorable response in $100 \%$ of their patients treated with $0.1 \%$ isotretinoin. The results recorded in relation to topical treatment clearly differ from our own, since we observed improvement in only one patient, with worsening in two subjects, and no changes in another three patients. These differences again could be explained by the different evolutive pattern of leukoplakias compared with PVL.

Overall, when considering the use of retinoids for the treatment of PVL, the most significant findings are perhaps the absence of clinical resolution of the white lesions in any of our patients (in contrast to the findings of other authors in patients with leukoplakias unrelated to PVL), and the worsening seen in a percentage of our patients despite the treatment provided (this not being observed or at least not reported by other authors who have used retinoids to treat leukoplakia).

The mean time of leukoplakia evolution towards oral squamous cell carcinoma varies in the literature from 4.4 years according to Bagán et al. (3) to 11.6 years as documented by Silverman and Gorsky (6). The time from first carcinoma to successive carcinomas in PVL patients has only been documented by Bagán et al., who recorded a mean time from first to second oral squamous cell carcinoma of $19.20 \pm 13.41$ months, and a mean time from second to third carcinoma of $17.47 \pm 6.63$ months. Lodi et al (17) in a systematic review about treatment of leukoplakias concluded that none of the therapies evaluated (including retinoids) offered benefit versus placebo in preventing the malignant transformation of leukoplakia. In our study, the appearance of two carcinomas, one of them during the period of treatment and the other in the immediate posterior period, supports the hypothesis that retinoids do not modify the natural evolution of PVL to oral squamous cell carcinomas.

A particularly relevant aspect of treatment with retinoids is its important toxicity, especially when admin- istered via the systemic route. All our patients who received systemic retinoids suffered adverse effects associated to treatment, while in contrast only one patient in the topical treatment group experienced problems in the form of itching in the oral cavity. The most frequent adverse effects cheilitis, desquamation, pruritus, alopecia and rhinitis. Most of the adverse effects recorded in our series coincided with the known side effects of retinoids (pruritus, skin desquamation, alopecia, fragile nails, triglyceride alterations, etc). However, two of the patients suffered serious adverse effects not described in the Summary of Product Characteristics of the medication used. In effect, after three months of treatment, one of the patients administered $25 \mathrm{mg}$ of acitretin/day developed intense rectal bleeding and cramps of the extremities that made standing and walking difficult. Suppression of the drug led to resolution of these manifestations. Another patient with the same treatment developed an asthmatic crisis related to acitretin.

In conclusion, although topical or systemic retinoic acid offers some degree of improvement in a little over one-third of all patients with PVL, the large proportion of individuals who worsen despite treatment, and the high frequency of adverse effects, make it necessary to conduct experimental studies before indicating or contraindicating the use of retinoids for the treatment of proliferative verrucous leukoplakia.

\section{References}

1. Hansen LS, Olson JA, Silverman S Jr. Proliferative verrucous leukoplakia. A long-term study of thirty patients. Oral Surg Oral Med Oral Pathol. 1985;60:285-98.

2. Cabay RJ, Morton TH Jr, Epstein JB. Proliferative verrucous leukoplakia and its progression to oral carcinoma: a review of the literature. J Oral Pathol Med. 2007;36:255-61.

3. Bagán JV, Murillo J, Poveda R, Gavaldá C, Jiménez Y, Scully C. Proliferative verrucous leukoplakia: unusual locations of oral squamous cell carcinomas, and field cancerization as shown by the appearance of multiple OSCCs. Oral Oncol. 2004;40:440-3.

4. Femiano F, Gombos F, Scully C. Oral proliferative verrucous leukoplakia (PVL); open trial of surgery compared with combined therapy using surgery and methisoprinol in papillomavirus-related PVL. Int J Oral Maxillofac Surg. 2001;30:318-22.

5. Zakrzewska JM, Lopes V, Speight P, Hopper C. Proliferative verrucous leukoplakia: a report of ten cases. Oral Surg Oral Med Oral Pathol Oral Radiol Endod. 1996;82:396-401.

6. Silverman S Jr, Gorsky M. Proliferative verrucous leukoplakia: a follow-up study of 54 cases. Oral Surg Oral Med Oral Pathol Oral Radiol Endod. 1997;84:154-7.

7. Bagan JV, Jimenez Y, Sanchis JM, Poveda R, Milian MA, Murillo $\mathrm{J}$, et al. Proliferative verrucous leukoplakia: high incidence of gingival squamous cell carcinoma. J Oral Pathol Med. 2003;32:379-82.

8. Orfanos CE, Zouboulis CC, Almond-Roesler B, Geilen CC. Current use and future potential role of retinoids in dermatology. Drugs. 1997;53:358-88.

9. Hong WK, Endicott J, Itri LM, Doos W, Batsakis JG, Bell R, et al. 13-cis-retinoic acid in the treatment of oral leukoplakia. N Engl J Med. 1986;315:1501-5.

10. Silverman S Jr, Renstrup G, Pindborg JJ. Studies In Oral Leukoplakias. VII. Further Investigations On The Effects Of Vitamin A On Keratinization. Acta Odontol Scand. 1963;21:553-70. 
11. Sankaranarayanan R, Mathew B, Varghese C, Sudhakaran PR, Menon V, Jayadeep A, et al. Chemoprevention of oral leukoplakia with vitamin A and beta carotene: an assessment. Oral Oncol. 1997;33:231-6.

12. Shah JP, Strong EW, DeCosse JJ, Itri L, Sellers P. Effect of retinoids on oral leukoplakia. Am J Surg. 1983;146:466-70.

13. Epstein JB, Gorsky M. Topical application of vitamin A to oral leukoplakia: A clinical case series. Cancer. 1999;86:921-7.

14. Boisnic S, Licu D, Ben Slama L, Branchet-Gumila MC, Szpirglas H, Dupuy P. Topical retinaldehyde treatment in oral lichen planus and leukoplakia. Int J Tissue React. 2002;24:123-30.

15. Scardina GA, Carini F, Maresi E, Valenza V, Messina P. Evaluation of the clinical and histological effectiveness of isotretinoin in the therapy of oral leukoplakia: ten years of experience: is management still up to date and effective? Methods Find Exp Clin Pharmacol. 2006;28:115-9.

16. Piattelli A, Fioroni M, Santinelli A, Rubini C. Bcl-2 expression and apoptotic bodies in 13-cis-retinoic acid (isotretinoin)-topically treated oral leukoplakia: a pilot study. Oral Oncol. 1999;35:314-20.

17. Lodi G, Sardella A, Bez C, Demarosi F, Carrassi A. Interventions for treating oral leukoplakia. Cochrane Database Syst Rev. 2006;4:CD001829. 